\title{
Is There a Link between COVID-19 and Periodontal Disease? A Narrative Review
}

\author{
Andreas Grigoriadis ${ }^{1,20}$ Ismo T. Räisänen ${ }^{3}$ Pirjo Pärnänen ${ }^{3}$ Taina Tervahartiala ${ }^{3}$ Timo Sorsa ${ }^{3,4}$ \\ Dimitra Sakellari ${ }^{1}$
}

${ }^{1}$ Department of Preventive Dentistry, Periodontology and Implant

Address for correspondence Andreas Grigoriadis, DDS, MS, PhD, Biology, Dental School, Aristotle University of Thessaloniki, Thessaloniki, Greece

2 Department of Periodontology, 424 General Military Training Hospital, Thessaloniki, Greece

${ }^{3}$ Department of Oral and Maxillofacial Diseases, Head and Neck Center, University of Helsinki and Helsinki University Hospital, Helsinki, Finland

${ }^{4}$ Department of Dental Medicine, Division of Periodontology, Karolinska Institutet, Stockholm, Sweden Department of Preventive Dentistry, Periodontology and Implant Biology, Dental School, Aristotle University, Thessaloniki, Greece (e-mail: andreasgrigor@gmail.com).

Eur J Dent 2022;16:514-520.

\begin{abstract}
The coronavirus disease 2019 (COVID-19) pandemic greatly affected human wellbeing, social behavior, global economy, and healthcare systems. Everyday clinical practice in dentistry has been adjusted to the increased hazards of aerosol production by routine dental procedures. The objective of this study was to assess the existing literature to determine possible mechanisms of a relationship between COVID-19 and periodontitis, as well as describe findings from relevant epidemiological studies.

Scarce data exist in the literature that directly addresses the relationship between the two diseases. However, several data describe the role of the oral cavity and periodontal tissues as portals of entry of severe acute respiratory syndrome-coronavirus-2 (SARS(oV-2), and the contribution of cytokines known to be produced in periodontal disease to severe forms of COVID-19. It is also suggested from the current literature that

\section{Keywords}

- coronavirus disease 2019

- periodontitis

- periodontal disease

- oral health

- oral-general health periodontal disease, shown to be associated with systemic diseases such as diabetes mellitus, cardiovascular and respiratory diseases, shares common risk factors withespecially-severe forms of COVID-19.

Further clinical studies are required to establish the relationship between these diseases. Oral hygiene performance and intact periodontal tissues can assist in mitigating the pandemic, and it is suggested that dental practitioners can contribute to identifying at-risk patients.
\end{abstract}

\section{Introduction}

Severe acute respiratory syndrome-coronavirus-2 (SARSCoV-2) has caused a pandemic with immense impact on human well-being, social behavior, global economy, and certainly, healthcare systems. As of July 5, 2021, there have been 183,560,151 confirmed cases of COVID-19, including 3,978,581 deaths reported to the World Health Organization (WHO), with the Americas and Europe reporting at least 128 million people affected by the disease. ${ }^{1}$ published online January 6, 2022
DOI https://doi.org/ 10.1055/s-0041-1740223. ISSN 1305-7456.

\section{(C) 2022. The Author(s).}

This is an open access article published by Thieme under the terms of the Creative Commons Attribution License, permitting unrestricted use, distribution, and reproduction so long as the original work is properly cited. (https://creativecommons.org/licenses/by/4.0/)

Thieme Medical and Scientific Publishers Pvt. Ltd., A-12, 2nd Floor, Sector 2, Noida-201301 UP, India 
The genome of this novel coronavirus has been identified and fully described in early 2020, triggering a worldwide scientific effort to confront this new challenge. ${ }^{2,3}$

COVID-19 presents various clinical signs, with most patients exhibiting mild symptoms including fever, dry cough, anosmia, ageusia, and fatigue. ${ }^{4}$ Unfortunately, up to $20 \%$ of patients develop severe disease, which leads to hospitalization and possibly necessitates intensive care unit (ICU) treatment, with grave-even fatal-complications, such as acute respiratory distress syndrome (ARDS), multiorgan failures, and sepsis. ${ }^{4}$

Despite their similarities regarding structure, pathways of transmission, and clinical symptoms, a higher infectious rate of SARS-CoV-2 compared with SARS-CoV-1 and Middle East Respiratory Syndrome-CoV (MERS-CoV) has been reported, contributing to the global spread and raising substantial concerns for controlling the transmission of the infection., 5

Transmission of SARS-CoV-2 occurs mainly via respiratory droplets expelled by talking, sneezing, coughing from face-to-face contact and, to a lesser degree, via contaminated surfaces, and it is estimated that up to $59 \%$ of all transmissions came from the asymptomatic transmission, comprising 35\% from presymptomatic subjects, and $24 \%$ from subjects who never develop symptoms. ${ }^{7}$

Of particular concern for the dental community is that aerosol spread of the virus can occur. ${ }^{8}$ Several dental procedures in clinical practice generate aerosol, and the close contact of the dental practitioner with the patient leads to an increased risk of transmission. Consequently, the implementation of routine dental procedures has been affected worldwide, with aerosol-generating procedures generally suspended, practice limited to emergency cases, and the introduction of teledentistry-when feasible. ${ }^{9,10}$

One year after the onset of the pandemic, various treatments have been proposed -and tested for the disease, mostly under pressure for treating patients at risk for severe clinical symptomatology. ${ }^{11}$

A great hope for mitigation of the pandemic has been the development of safe and effective vaccines against SARSCoV-2. Due to the collaboration of the scientific community, pharmaceutical companies, and health organizations globally, certain vaccines are already authorized for human use, with a substantial number under clinical trials. ${ }^{12}$ As of July 5 , 2021 , it is estimated that $2,988,941,529$ vaccine doses have been administered. ${ }^{1}$

Of particular interest for the dental community is the possible relationship between COVID-19 and periodontal disease.

Therefore, the purpose of the present narrative review was to discuss scientific information on the role of the oral cavity and periodontal tissues as portals of entry of SARSCoV-2, the connection of risk factors for COVID-19 with periodontal disease, and the biological plausibility of this relationship.

\section{Methods}

A comprehensive search was performed in three electronic databases (MEDLINE/PubMed, Scopus, Cochrane Controlled register of Trials) from January 2020 until February 2021. Manual searching was also performed on Google and Google Scholar. Only articles written in English were considered initially. The following keywords were used in the search: "periodontal disease," “COVID-19," "SARS-CoV-2," "oral cavity," “oral hygiene," "ACE2 receptors," "periodontitis risk factors," and "COVID-19 risk factors." All types of articles considered relevant for discussion were selected and analyzed in detail. The reference lists of the selected articles were then hand-searched for additional articles deemed appropriate by two of the authors (AG and DS). The literature search located 193 studies. After duplicate removal, 81 studies were assessed based on the title and abstract. Of them, 66 studies were examined as full-text articles. Twentyseven articles and information from official Web sites describing the epidemiology, transmission, pathophysiology, and treatment of COVID-19 were also included in the present review.

\section{Interrelation between SARS-CoV-2 and the Oral Cavity} Of pivotal importance for the comprehension of SARS-CoV-2 pathogenesis was recognizing the specific receptors utilized by the virus to invade host cells. It has been shown that the Angiotensin-Converting Enzyme -2 (ACE-2) consists of a major route for viral entry to the cells, similarly to SARSCoV-1. ${ }^{13,14}$ In addition, it was reported that the affinity of binding of the viral spike (S) protein is 10 to 20 fold increased compared with SARS-CoV-1, and this fact could explain the highly contagious ability of this specific virus. ${ }^{14,15}$ ACE2 is not the only molecule promoting viral invasiveness. Transmembrane protease serine 2 (TMPRSS2) and furin are also described as cell entry assisting molecules for SARS-CoV-2, albeit to a lesser extent. ${ }^{13}$ ACE-2 is distributed throughout several tissues, and its' expression and function are affected by several factors, including genetic and demographic characteristics, lifestyle habits, comorbidities, and concurrent medications. ${ }^{14}$ It is already known that ACE2 is highly expressed on epithelial cells of principal target organs of SARS-CoV-2 such as the lungs, kidneys, and intestines. ${ }^{14,16}$

\section{These Receptors are Expressed in the Oral Cavity}

According to the report of Xu et al, ACE2 is expressed on the epithelial cells of various areas of the oral mucosa, particularly on the tongue compared with buccal or gingival cells, thus implicating the oral cavity as a highly susceptible environment for SARS-CoV-2 infection. ${ }^{6}$ These findings were further enhanced by consequent studies, applying a variety of techniques, in which it has been shown that enriched ACE2 expression is observed apart from the surface of the tongue in several areas of the head and neck region. Examples of those areas are the oral cavity epithelial cells (including the gingival sulcus/periodontal pocket epithelium), the salivary glands, the vocal cords, and the sinuses. ${ }^{17-19}$

ACE2 is also identified in patients with periodontal disease in various cell types, such as fibroblasts, osteoblasts, and osteoclasts, which are critical for homeostasis for hard and soft tissues around teeth and implants. ${ }^{20,21}$ Earlier studies in 
animals have also demonstrated ACE2 expression in gingival fibroblasts. $^{22}$ Therefore, it is suggested that this line of research will elucidate methods of viral transmission leading to preventive strategies and explain aspects of the pathophysiology of COVID-19. For example, the ACE2 (TMPRSS2) and abundant furin expression on taste bud-derived cells cultures might explain the olfactory abnormality, one of the known causes of dysgeusia in COVID-19. ${ }^{17}$ Based on these findings, several authors have formulated a hypothesis that the oral environment, including gingival sulci/periodontal pockets ${ }^{23,24}$ and salivary glands, ${ }^{25}$ can serve as potential reservoirs for shedding SARS-CoV-2. Epithelial cells of salivary glands have previously been shown to be infected in studies regarding SARS-CoV-1 and were considered a major source for the virus in saliva, ${ }^{26}$ and the same may apply for SARS-CoV-2. The presence of increased viral load in saliva samples and the recently reported detection of viral RNA in gingival crevicular fluid (GCF) also demonstrate the connection between COVID-19 disease and the oral cavity. ${ }^{27-30}$ Due to this fact, saliva sampling and analysis for detecting SARSCoV-2 infection have been supported by several studies. In addition, saliva sampling and analysis offer ease convenience of patients and non-invasiveness and could serve as a diagnostic tool for large-scale screening, compared with the "gold-standard," the nasopharyngeal swab nuclear acid amplification testing (NAAT). Nevertheless, it should be noticed that findings in the literature regarding the diagnostic value of saliva sampling have not been unequivocal or provide similar performance to the gold standard. ${ }^{28,31,32}$ Results from a recently published systematic review and meta-analysis suggest that saliva NAAT diagnostic accuracy is similar to nasopharyngeal swab NAAT, especially in the ambulatory setting, suggesting larger-scale research on its' alternative use. ${ }^{33}$ In line with these findings, the Federal Drug Administration (FDA) has authorized SARS-CoV-2 testing on saliva samples collected at home using a self-collection kit in the United States Federal Drug Administration. ${ }^{34}$ Findings from a May 2021 study investigating SARS-CoV-2 infection of the oral cavity and saliva have strongly confirmed that the oral cavity is an important site for infection from the virus and that saliva is suggested as a route for transmission. ${ }^{35}$

Although the data mentioned above describe the oral environment as biologically favorable for SARS-CoV-2 invasion, proliferation, and shedding source, one should keep in mind that it also consists of an effective barrier against microbial pathogens. Effective innate immunity mechanisms and locally produced molecules such as the defensins constitute a well-documented first line of defense that might not allow for colonization and proliferation of the virus. These properties may account for controversial results reported in the literature. ${ }^{36,37}$

\section{Risk Factors for COVID-19 and Periodontal Disease}

Several risk factors have emerged following the surge and spread of COVID-19, especially for cases with severe complications and are still under investigation. According to the Centers for Disease Control (USA), the following underlying medical conditions consist risk factors for developing severe COVID-19: cancer, chronic kidney disease, chronic obstructive pulmonary disease (COPD), Down's syndrome, heart conditions, such as heart failure, coronary artery disease or cardiomyopathies, solid organ transplants, obesity (body mass index of $30 \mathrm{~kg} / \mathrm{m}^{2}$ or higher), pregnancy, sickle cell disease, smoking, and type 2 diabetes. ${ }^{38}$

Among them, certain conditions and clinical entities are strongly related to periodontal disease. Comorbidities, as reported so far for COVID-19, are also known to affect the onset, extent, and severity of periodontal diseases, and this relationship is worthy of investigating since it could positively affect the recognition and early intervention of at-risk individuals.

For example, age $>65$ years is considered a significant risk factor for severe COVID-19, probably due to comorbidities and the reduced immune response in this age group, and it is also suggested that male subjects are also more prone to complications of the disease. ${ }^{39}$ Age is an established risk factor for periodontal disease, ${ }^{40}$ providing a link for dental practitioners to emphasize the need for optimal oral hygiene and healthy periodontal tissues to older patients to reduce complications of both disease entities similarly to other chronic diseases.

Obesity is also reported to increase the risk for severe COVID-19 disease, probably due to reduced respiratory function and amplified inflammatory response. The presence of a "primed" inflammatory status with increased production of pro-inflammatory cytokines and reactive oxygen species in obese individuals has also been suggested as a risk factor for the development of periodontal disease. ${ }^{41}$

This is also the case for the link between cardiovascular and periodontal disease, a relationship widely documented in the literature at the biological and epidemiological levels. $^{42}$ Comorbidities such as heart failure, coronary artery disease, cardiomyopathies, and pulmonary hypertension are currently considered risk factors for severe COVID-19 disease. In contrast, other cardiovascular or cerebrovascular diseases, such as hypertension or stroke, might increase the risk of severe illness from the disease. ${ }^{38}$

A plausible mechanism of the interplay between periodontitis, the above-mentioned cardiovascular conditions, and COVID-19, is the increase of systemic inflammation induced by diseased periodontal tissues with over-production of pro-inflammatory mediators such as cytokines, both locally and systemically. A "cytokine storm," as described below, is a common finding in severe COVID-19 infections, suggesting a possible exacerbation when periodontal disease is present in the oral cavity.

Chronic obstructive pulmonary disease (COPD) refers to a group of diseases that cause airflow blockage and breathingrelated problems, including emphysema and chronic bronchitis. The association between periodontitis and respiratory diseases (asthma, COPD, and pneumonia) has a strong biological background based on the potential role of oral bacteria to facilitate infection from respiratory pathogens. This is also strongly suggested by epidemiological findings. ${ }^{43,44}$ Recent studies have shown that severe periodontal disease 
increases the risk for COPD mortality in older patients, and on the other hand, in subjects over 70 years, enhanced oral hygiene can prevent airway infections. ${ }^{45,46}$ Given the strong relation of severe COVID-19 to COPD, dental practitioners could thus be helpful to identify risk groups among patients exhibiting COPD.

The bidirectional linking of periodontal disease and diabetes is well established in the literature. ${ }^{47}$ Data from recent large-scale clinical studies have shown that risks of fatal or intensive care unit treated COVID-19 cases were elevated in patients with diabetes mellitus types 1 and 2 , or even admission hyperglycemia regardless of prior diagnosis of diabetes. ${ }^{48,49}$ Hyperglycemia is known to dysregulate the immune response, promote the infection of cells from SARSCoV-2 by glycosylation of ACE2, and elevate the gene expression of matrix-metalloproteinases which promote inflammation.

\section{Biological Plausibility and Review of the Literature}

The SARS-CoV-2 infection triggers a profound cytokine response in the host, often referred to as a 'cytokine storm' comprising a series of mediators important for inflammation but not assisting ineffective virus clearance. ${ }^{50}$

This 'cytokine storm' is exhibited as elevated serum levels of TNF- $\alpha$, IL-1 $\beta$, IL-6, IL-8, G-CSF and GM-CSF, and chemokines, such as MCP1, IP10, and MIP1 $\alpha$, with higher levels in those who are critically ill. Elevated Th17 pathway responses could lead to severe pulmonary edema and tissue damage in lung infections and have also been observed in patients of SARS-CoV- 1 and MERS-CoV. ${ }^{50-53}$

It is known that targeting the cytokines mentioned above with specific antibodies such as sarilumab and tocilizumab is an effective pharmacological approach for immune-mediated inflammatory diseases (IMIDs), such as rheumatoid arthritis (RA), spondyloarthritis (SpA), and Crohn's disease. ${ }^{11}$ The inhibition of cytokines might be considered inducing immune suppression, but these molecules are known to neutralize specific mediators, therefore attenuating the hyperinflammatory reaction to the SARS-CoV-2 infection and not inducing a generalized and harmful immune suppression. ${ }^{50}$ Therefore, monoclonal antibodies directed against key inflammatory cytokines consist of a potential class of adjunctive therapies for COVID-19 under investigation. ${ }^{11}$

Of interest is the fact that ACE2 functions as a down-regulator of the inflammatory response by blocking oxidative stress, cell proliferation, and the release of pro-inflammatory mediators. In COVID-19 patients, a reduction of ACE2 levels is observed due to the complexes formed between the virus and these proteins, and this fact might also contribute to the "cytokine storm" observed, especially in severe cases of the disease. ${ }^{54}$

Similarly, immune-mediated pathogenetic pathways have also been shown for periodontitis, and the cytokines produced locally in the periodontal tissues are implicated in the interaction mechanism of periodontal inflammation and systemic diseases. ${ }^{55}$ This fact supports the hypothesis that meticulous oral hygiene and intact, non-inflamed periodon- tal tissues can prevent COVID-19 complications, at least in certain patient groups.

Matrix metalloproteinases (MMPs) are involved in tissue destructive oral diseases such as periodontitis, peri-implantitis, dental caries, and oral cancer. ${ }^{56}$ MMP-8, also known as neutrophil collagenase, causes unwanted tissue destruction in periodontitis/peri-implantitis due to increased activation of MMP-8 inactive periodontitis/peri-implantitis, ${ }^{57}$ which has also been demonstrated to be reflected in oral fluids in vivo. ${ }^{58}$ Furthermore, previous studies have shown an association between elevated levels of active MMP-8 (aMMP-8) in oral fluids and clinical periodontal parameters. ${ }^{59-62}$ In addition, diabetes is known to elevate and activate MMPs in gingival tissues, and oral fluids and recent studies have shown the usefulness of aMMP- 8 point-of-care testing in the clinic concerning the identification of patients at risk of prediabetes, gestational diabetes, and progression of periodontitis in HNC radiotherapy. ${ }^{63-66}$ Given the ability of the test to conveniently identify people with diabetes with periodontitis, the possibility to identify COVID-risk patients such as the populations mentioned above is eventually exerted.

The relationship between periodontitis and systemic diseases and the link between them and the active form of periodontitis, reflected as elevated aMMP-8 levels in oral fluids, makes the aMMP-8 point-of-care testing an interesting tool for future COVID-19 studies. ${ }^{67}$

The possible coexistence of COVID-19 and periodontitis and the impact of oral hygiene could be a critical situation to observe to minimize severe manifestation of the disease and assist in identifying people at risk in the dental office.

In fact, according to the existing literature, several authors have, so far, underlined this possible interaction, the biological plausibility, and mechanisms and implications for clinical practice of the interplay between COVID-19 and periodontitis. $^{68-73}$

Scarce data from clinical trials in the current literature exist regarding the impact of periodontal disease in severe COVID-19 cases. In a study with more than 13.000 participants in the United Kingdom, 12\% were COVID-19 positive, self-reported painful or bleeding gums, and loose teeth were used as surrogate markers for periodontal disease. ${ }^{74}$ According to the study's findings, the risk of COVID-19 infection in participants with painful or bleeding gums and loose teeth compared with controls was not increased, while COVID-19 positive participants with painful or bleeding gums had a higher risk of mortality but not hospital admission. No correlation was found between self-reported loss of teeth and higher risk of hospital admission or mortality than the control group. Although evidence from this study is inconclusive, it should be emphasized that, according to findings, amongst the COVID-19 positive, there was significantly higher mortality for participants with periodontal disease. This line of research with clinical assessments of periodontal disease and the combination of dental and medical records, especially in hospital settings, will certainly improve our current understanding of the interplay between these diseases. 
A recently published case-control study, involving 568 patients in Qatar diagnosed with COVID-19, has shown that periodontitis was associated with 3.5 times higher risk of admission to ICU, 4.5 times need for mechanical ventilation, and almost nine times for death in Covid-19 patients, after adjusting for age, sex, BMI, smoking status, and other conditions. These findings were based on electronic medical and dental data and, albeit the limitations of the study (it involved only diagnostic radiographic criteria and was not designed to address causality), it is suggested that establishing and maintaining periodontal health may become an important part of the care of these patients. ${ }^{75}$

It has also been suggested that the relationship between the oral microbiome and severe COVID-19 infection should be investigated to enlighten the outcomes of COVID-19 disease. The main comorbidities associated with an increased risk of severe, even fatal from COVID-19 as described above, are also associated with altered oral biofilms and periodontal disease. Hence, the link between poor oral health and COVID-19 complications is suggested. ${ }^{76}$ Future studies aiming at defining the microbiome in patients with both clinical entities could clarify the impact of periodontitis on COVID-19 infection and the clinical course of the disease. In the context of the pandemic, until the importance of oral hygiene and the presence of oral disease in the severity and mortality risk of COVID-19 is solidly proven, the maintenance and optimization of oral hygiene should be emphasized to dental patients to target the reduction of the viral load in the oral cavity. In the same context, in anticipation of well-designed pre-clinical and clinical trials, the adjunctive use of various mouth rinses (mainly cetylpyridinium chloride, povidone-iodine, or chlorhexidine and hydrogen peroxide) has also been suggested, both as single preprocedural use and as daily use during a limited period. ${ }^{77}$

\section{Conclusions}

The surge of the COVID-19 pandemic is a challenge for periodontology. Exploring the mechanisms of interplay between COVID-19 and periodontal disease can contribute to the identification of patients at risk for complications and mitigate the spread and symptoms of COVID-19 by controlling periodontal inflammation and improving oral hygiene and health.

\section{Conflict of Interest}

T.S. is the inventor of US-patent $10488415 \mathrm{~B} 2$ and a Japanese patent 2016-554676. Rest authors declare no conflict of interest.

\section{References}

1 COVID-19 situation in the WHO European Region. Available at: https:// who.maps.arcgis.com/apps/dashboards/ead3c6475654481ca5 1c248d52ab9c61

2 Lu R, Zhao X, Li J, et al. Genomic characterisation and epidemiology of 2019 novel coronavirus: implications for virus origins and receptor binding. Lancet 2020;395(10224):565-574

3 Zhang Y-Z, Holmes EC. A genomic perspective on the origin and emergence of SARS-CoV-2. Cell 2020;181(02):223-227
4 Wiersinga WJ, Rhodes A, Cheng AC, Peacock SJ, Prescott HC. Pathophysiology, transmission, diagnosis, and treatment of coronavirus disease 2019 (COVID-19): a review. JAMA 2020;324(08): 782-793

5 Abdelrahman Z, Li M, Wang X. Comparative review of SARS-CoV2, SARS-CoV, MERS-CoV, and influenza a respiratory viruses. Front Immunol 2020;11:552909

6 Hu T, Liu Y, Zhao M, Zhuang Q, Xu L, He Q. A comparison of COVID19, SARS and MERS. PeerJ 2020;8:e9725

7 Johansson MA, Quandelacy TM, Kada S, et al. SARS-CoV-2 transmission from people without COVID-19 symptoms. JAMA Netw Open 2021;4(01):e2035057-e2035057

8 Peng X, Xu X, Li Y, Cheng L, Zhou X, Ren B. Transmission routes of 2019-nCoV and controls in dental practice. Int J Oral Sci 2020;12 (01):9

9 Guidance for dental settings. Accessed Nov 8, 2021 at. https:// www.cdc.gov/coronavirus/2019-ncov/hcp/dental-settings.html

10 EFP suggestions for the management of a dental clinic during the Covid-19 pandemic. Accessed Nov 8, 2021 at: https://www.efp.org/fileadmin/uploads/efp/Documents/covid19SafetyProtocol.pdf

11 Sanders JM, Monogue ML, Jodlowski TZ, Cutrell JB. Pharmacologic treatments for coronavirus disease 2019 (COVID-19): a review. JAMA 2020;323(18):1824-1836

12 COVID-19 vaccines. Accessed Nov 8, 2021 at: https://www.who.int/emergencies/diseases/novel-coronavirus-2019/covid-19vaccines

13 Hoffmann M, Kleine-Weber H, Schroeder S, et al. SARS-CoV-2 cell entry depends on ACE2 and TMPRSS2 and is blocked by a clinically proven protease inhibitor. Cell 2020;181(02):271-280.e8

14 Bourgonje AR, Abdulle AE, Timens W, et al. Angiotensin-converting enzyme 2 (ACE2), SARS-CoV-2 and the pathophysiology of coronavirus disease 2019 (COVID-19). J Pathol 2020;251(03): 228-248

15 Wrapp D, Wang N, Corbett KS, et al. Cryo-EM structure of the 2019-nCoV spike in the prefusion conformation. Science 2020; 367(6483):1260-1263

16 Hamming I, Timens W, Bulthuis ML, Lely AT, Navis G, van Goor H. Tissue distribution of ACE2 protein, the functional receptor for SARS coronavirus. A first step in understanding SARS pathogenesis. J Pathol 2004;203(02):631-637

17 Sakaguchi W, Kubota N, Shimizu T, et al. Existence of SARS-CoV2 entry molecules in the oral cavity. Int J Mol Sci 2020;21(17): 6000

18 Descamps G, Verset L, Trelcat A, et al. ACE2 protein landscape in the head and neck region: the conundrum of SARS-CoV-2 infection. Biology (Basel) 2020;9(08):235

19 Wu C, Zheng M. Single-cell RNA expression profiling shows that ACE2, the putative receptor of COVID-2019, has significant expression in nasal and mouth tissue, and is co-expressed with TMPRSS2 and not co-expressed with SLC6A19 in the tissues. Preprints 2020. Doi: 10.21203/rs.3.rs-16992/v1

20 Gabriele LG, Morandini AC, Dionísio TJ, Santos CF. Angiotensin II type 1 receptor knockdown impairs interleukin-1 $\beta$-induced cytokines in human periodontal fibroblasts. J Periodontol 2017;88 (01):e1-e11

21 Santos CF, Morandini AC, Dionísio TJ, et al. Functional local reninangiotensin system in human and rat periodontal tissue. PLoS One 2015;10(08):e0134601

22 Santos CF, Akashi AE, Dionísio TJ, et al. Characterization of a local renin-angiotensin system in rat gingival tissue. J Periodontol 2009;80(01):130-139

23 Badran Z, Gaudin A, Struillou X, Amador G, Soueidan A. Periodontal pockets: a potential reservoir for SARS-CoV-2? Med Hypotheses 2020;143:109907

24 Kheur S, Kheur M, Gupta AA, Raj AT. Is the gingival sulcus a potential niche for SARS-corona virus-2? Med Hypotheses 2020; 143:109892 
25 Xu J, Li Y, Gan F, Du Y, Yao Y. Salivary glands: potential reservoirs for COVID-19 asymptomatic infection. J Dent Res 2020;99(08): 989-989

26 Liu L, Wei Q, Alvarez X, et al. Epithelial cells lining salivary gland ducts are early target cells of severe acute respiratory syndrome coronavirus infection in the upper respiratory tracts of rhesus macaques. J Virol 2011;85(08):4025-4030

27 Wyllie AL, Fournier J, Casanovas-Massana A, et al. Saliva or nasopharyngeal swab specimens for detection of SARS-CoV-2. N Engl J Med 2020;383(13):1283-1286

28 Gupta S, Mohindra R, Chauhan P, et al. SARS-CoV-2 detection in gingival crevicular fluid. J Dent Res 2021;100(02):187-193

29 To KKW, Yip CCY, Lai CYW, et al. Saliva as a diagnostic specimen for testing respiratory virus by a point-of-care molecular assay: a diagnostic validity study. Clin Microbiol Infect 2019;25(03): 372-378

30 Zhu J, Guo J, Xu Y, Chen X. Viral dynamics of SARS-CoV-2 in saliva from infected patients. J Infect 2020;81(03):e48-e50

31 Jamal AJ, Mohammad M, Coomes E, et al. Sensitivity of nasopharyngeal swabs and saliva for the detection of severe acute respiratory syndrome coronavirus 2 (SARS-CoV-2). medRxiv 2020. Doi: $10.1101 / 2020.05 .01 .20081026$

32 Pasomsub E, Watcharananan SP, Boonyawat K, et al. Saliva sample as a non-invasive specimen for the diagnosis of coronavirus disease 2019: a cross-sectional study. Clin Microbiol Infect 2021;27(02):285.e1-285.e4

33 Butler-Laporte G, Lawandi A, Schiller I, et al. Comparison of Saliva and Nasopharyngeal Swab Nucleic Acid Amplification Testing for Detection of SARS-CoV-2: A Systematic Review and Meta-analysis. JAMA Intern Med.

34 Coronavirus (COVID-19) Update: FDA Authorizes First Diagnostic Test Using At-Home Collection of Saliva Specimens. Accessed Nov 8, 2021 at: https://www.fda.gov/news-events/press-announcements/coronavirus-covid-19-update-fda-authorizes-first-diagnostic-test-using-home-collection-saliva

35 Huang N, Pérez P, Kato T, et al; NIH COVID-19 Autopsy Consortium HCA Oral and Craniofacial Biological Network. SARS-CoV-2 infection of the oral cavity and saliva. Nat Med 2021;27(05):892-903

36 Troeltzsch M, Berndt R, Troeltzsch M. Is the oral cavity a reservoir for prolonged SARS-CoV-2 shedding? Med Hypotheses 2021; 146:110419

37 Fernandes Matuck B, Dolhnikoff M, Maia GVA, et al. Periodontal tissues are targets for Sars-Cov-2: a post-mortem study. J Oral Microbiol 2020;13(01):1848135

38 People with Certain Medical Conditions. Accessed Nov 8, 2021 at. https://www.cdc.gov/coronavirus/2019-ncov/need-extra-precautions/people-with-medical-conditions.html

39 Jordan RE, Adab P, Cheng K. COVID-19: risk factors for severe disease and death. BMJ 2020. Doi: 10.1136/bmj.m1198

40 Persson GR. Periodontal complications with age. Periodontol 2000 2018;78(01):185-194

41 Suvan J, Harrington Z, Petrie A, et al. Obesity as predictive factor of periodontal therapy clinical outcomes: a cohort study. J Clin Periodontol 2020;47(05):594-601

42 Sanz M, Marco Del Castillo A, Jepsen S, et al. Periodontitis and cardiovascular diseases: Consensus report. J Clin Periodontol 2020;47(03):268-288

43 Gomes-Filho IS, Cruz SSD, Trindade SC, et al. Periodontitis and respiratory diseases: A systematic review with meta-analysis. Oral Dis 2020;26(02):439-446

44 Huffnagle GB, Dickson RP, Lukacs NW. The respiratory tract microbiome and lung inflammation: a two-way street. Mucosal Immunol 2017;10(02):299-306

45 Sjögren P, Nilsson E, Forsell M, Johansson O, Hoogstraate J. A systematic review of the preventive effect of oral hygiene on pneumonia and respiratory tract infection in elderly people in hospitals and nursing homes: effect estimates and methodologi- cal quality of randomized controlled trials. J Am Geriatr Soc 2008; 56(11):2124-2130

46 Qian Y, Yuan W, Mei N, et al. Periodontitis increases the risk of respiratory disease mortality in older patients. Exp Gerontol 2020;133:110878

47 Sanz M, Ceriello A, Buysschaert M, et al. Scientific evidence on the links between periodontal diseases and diabetes: consensus report and guidelines of the joint workshop on periodontal diseases and diabetes by the International Diabetes Federation and the European Federation of Periodontology. J Clin Periodontol 2018;45(02):138-149

48 Carrasco-Sánchez FJ, López-Carmona MD, Martínez-Marcos FJ, et al; SEMI-COVID-19 Network. Admission hyperglycaemia as a predictor of mortality in patients hospitalized with COVID-19 regardless of diabetes status: data from the Spanish SEMI-COVID19 Registry. Ann Med 2021;53(01):103-116

49 McGurnaghan SJ, Weir A, Bishop J, et al.Public Health Scotland COVID-19 Health Protection Study Group Scottish Diabetes Research Network Epidemiology Group. Risks of and risk factors for COVID-19 disease in people with diabetes: a cohort study of the total population of Scotland. Lancet Diabetes Endocrinol 2021;9 (02):82-93

50 Schett G, Sticherling M, Neurath MF. COVID-19: risk for cytokine targeting in chronic inflammatory diseases? Nat Rev Immunol 2020;20(05):271-272

51 Wu D, Yang XO. TH17 responses in cytokine storm of COVID-19: An emerging target of JAK2 inhibitor Fedratinib. J Microbiol Immunol Infect 2020;53(03):368-370

52 Sahni V, Gupta S. COVID-19 \& Periodontitis: the cytokine connection. Med Hypotheses 2020;144:109908

53 Fabri GMC. Potential Link between COVID-19 and Periodontitis: Cytokine Storm, Immunosuppression, and Dysbiosis. Oral Health Dent Manag 2020;20:1-5

54 Mancini L, Quinzi V, Mummolo S, Marzo G, Marchetti E. Angiotensin-converting enzyme 2 as a possible correlation between COVID-19 and periodontal Disease. Appl Sci (Basel) 2020;10:6224

55 Cekici A, Kantarci A, Hasturk H, Van Dyke TE. Inflammatory and immune pathways in the pathogenesis of periodontal disease. Periodontol 2000 2014;64(01):57-80

56 Sorsa T, Tjäderhane L, Salo T. Matrix metalloproteinases (MMPs) in oral diseases. Oral Dis 2004;10(06):311-318

57 Sorsa T, Tjäderhane L, Konttinen YT, et al. Matrix metalloproteinases: contribution to pathogenesis, diagnosis and treatment of periodontal inflammation. Ann Med 2006;38(05):306-321

58 Lee W, Aitken S, Sodek J, McCulloch CA. Evidence of a direct relationship between neutrophil collagenase activity and periodontal tissue destruction in vivo: role of active enzyme in human periodontitis. J Periodontal Res 1995;30(01):23-33

59 Sorsa T, Ingman T, Suomalainen K, et al. Cellular source and tetracycline-inhibition of gingival crevicular fluid collagenase of patients with labile diabetes mellitus. J Clin Periodontol 1992;19 (02):146-149

60 Ryan ME, Ramamurthy NS, Sorsa T, Golub LM. MMP-mediated events in diabetes. Ann N Y Acad Sci 1999;878:311-334

61 Safkan-Seppälä B, Sorsa T, Tervahartiala T, Beklen A, Konttinen YT. Collagenases in gingival crevicular fluid in type 1 diabetes mellitus. J Periodontol 2006;77(02):189-194

62 Rathnayake N, Åkerman S, Klinge B, et al. Salivary biomarkers of oral health: a cross-sectional study. J Clin Periodontol 2013;40 (02):140-147

63 Grigoriadis A, Sorsa T, Räisänen I, Pärnänen P, Tervahartiala T, Sakellari D. Prediabetes/diabetes can be screened at the dental office by a low-cost and fast chair-side/point-of-care aMMP-8 immunotest. Diagnostics (Basel) 2019;9(04):151

64 Grigoriadis A, Räisänen IT, Pärnänen P, Tervahartiala T, Sorsa T, Sakellari D. Prediabetes/diabetes screening strategy at the periodontal clinic. Clin Exp Dent Res 2021;7(01):85-92 
65 Chaparro A, Realini O, Hernández M, et al. Early pregnancy levels of gingival crevicular fluid matrix metalloproteinases- 8 and -9 are associated with the severity of periodontitis and the development of gestational diabetes mellitus. J Periodontol 2021;92(02): 205-215

66 Keskin M, Lähteenmäki H, Rathnayake N, et al. Active matrix metalloproteinase- 8 and interleukin- 6 detect periodontal degeneration caused by radiotherapy of head and neck cancer: a pilot study. Expert Rev Proteomic 2021;17(10): 777-784

67 Räisänen IT, Umeizudike KA, Pärnänen P, et al. Periodontal disease and targeted prevention using aMMP-8 point-of-care oral fluid analytics in the COVID-19 era. Med Hypotheses 2020;144:110276

68 Bertolini M, Pita A, Koo S, Cardenas A, Methil A. Periodontal disease in the COVID-19 era: potential reservoir and increased risk for SARS-CoV-2. Pesqui Bras Odontopediatria Clin Integr 2020;20(supp1):e0134https://doi.org/10.1590/pboci.2020.162

69 Sharma A, Chauhan S, Sharma R, Jindal V, Rajput P, Jindal V. How chronic periodontitis associated with systemic diseases increases the case fatality rate among COVID-19 Patients. J Adv Med Dent Scie Res 2020;8:71-77

70 Pitones-Rubio V, Chávez-Cortez EG, Hurtado-Camarena A, González-Rascón A, Serafín-Higuera N. Is periodontal disease a risk factor for severe COVID-19 illness? Med Hypotheses 2020; 144:109969

71 Siddharthan S, Naing NN, Wan-Arfah N. Periodontal disease and COVID 19. J Pharm Res Int 2020;32(32):88-91. Doi: 10.9734/JPRI/ 2020/v32i3230937

72 Kadkhodazadeh M, Amid R, Moscowchi A. Does COVID-19 affect periodontal and peri-implant diseases? J Long Term Eff Med Implants 2020;30(01):1-2

73 Madapusi Balaji T, Varadarajan S, Rao USV, et al. Oral cancer and periodontal disease increase the risk of COVID 19? A mechanism mediated through furin and cathepsin overexpression. Med Hypotheses 2020;144:109936

74 Larvin H, Wilmott S, Wu J, Kang J. The impact of periodontal disease on hospital admission and mortality during COVID-19 pandemic. Front Med (Lausanne) 2020;7:604980

75 Marouf N, Cai W, Said KN, et al. Association between periodontitis and severity of COVID-19 infection: a case-control study. J Clin Periodontol 2021;48(04):483-491

76 Sampson V, Kamona N, Sampson A. Could there be a link between oral hygiene and the severity of SARS-CoV-2 infections? Br Dent J 2020;228(12):971-975

77 Herrera D, Serrano J, Roldán S, Sanz M. Is the oral cavity relevant in SARS-CoV-2 pandemic? Clin Oral Investig 2020;24(08):2925-2930 